\title{
Asymmetry Index on Marginal Homogeneity for Square Contingency Tables with Ordered Categories
}

\author{
Kouji Tahata, Kanau Kawasaki, Sadao Tomizawa \\ Department of Information Sciences, Faculty of Science and Technology, Tokyo University of Science, \\ Chiba, Japan \\ Email: \{kouji_tahata, tomizawa\}@is.noda.tus.ac.jp, k.kawasaki123@gmail.com
}

Received December 27, 2011; revised January 25, 2012; accepted February 10, 2012

\begin{abstract}
For square contingency tables with ordered categories, the present paper considers two kinds of weak marginal homogeneity and gives measures to represent the degree of departure from weak marginal homogeneity. The proposed measures lie between -1 to 1 . When the marginal cumulative logistic model or the extended marginal homogeneity model holds, the proposed measures represent the degree of departure from marginal homogeneity. Using these measures, three kinds of unaided distance vision data are analyzed.
\end{abstract}

Keywords: Marginal Homogeneity; Marginal Cumulative Logistic Model; Measure; Square Contingency Table

\section{Introduction}

Consider an $R \times R$ square contingency table with ordered categories. Let $p_{i j}$ denote the probability that an observation will fall in the $i$ th row and $j$ th column of the table $(i=1, \cdots, R ; j=1, \cdots, R)$. Also let $X$ and $Y$ denote the row and column variables, respectively. The marginal homogeneity (MH) model ([1]) is defined by

$$
F_{i}^{X}=F_{i}^{Y} \text { for } i=1, \cdots, R-1
$$

where

$$
F_{i}^{X}=\sum_{k=1}^{i} p_{k \cdot}, \quad F_{i}^{Y}=\sum_{k=1}^{i} p_{\cdot k}
$$

with

$$
p_{k \cdot}=\sum_{t=1}^{R} p_{k t}, \quad p_{\cdot k}=\sum_{s=1}^{R} p_{s k} .
$$

When the MH model does not hold, we are interested in applying the model that has weaker restriction than the MH model. As such a model, for example, there are the marginal cumulative logistic (ML) model ([2]) and the extended marginal homogeneity (EMH) model ([3-5]). We are also interested in considering the other structure of weak MH. The measures to represent the degree of departure from $\mathrm{MH}$ are given by, for example, [6,7]. When the structure of weak MH does not hold, we are interested in measuring what degree the departure from weak $\mathrm{MH}$ is.

The present paper considers two kinds of structures of weak $\mathrm{MH}$ and proposes the measures to represent the degree of departure from weak $\mathrm{MH}$.

\section{Weak Marginal Homogeneity I and Measure}

\subsection{Submeasure I}

Let

$$
\Delta_{1}=\sum_{i=1}^{R-1}\left(F_{i}^{X}+F_{i}^{Y}\right)
$$

and

$$
F_{1(i)}^{*}=\frac{F_{i}^{X}}{\Delta_{1}}, \quad F_{2(i)}^{*}=\frac{F_{i}^{Y}}{\Delta_{1}}, \text { for } i=1, \cdots, R-1 .
$$

Note that $\sum_{i=1}^{R-1}\left(F_{1(i)}^{*}+F_{2(i)}^{*}\right)=1$. Assuming that $\left\{F_{i}^{X}+F_{i}^{Y} \neq 0\right\}$, consider the submeasure defined by

$$
\Psi_{1}=\frac{4}{\pi} \sum_{i=1}^{R-1}\left(F_{1(i)}^{*}+F_{2(i)}^{*}\right)\left(\theta_{i}^{(1)}-\frac{\pi}{4}\right),
$$

where

$$
\theta_{i}^{(1)}=\sin ^{-1}\left(\frac{F_{i}^{Y}}{\sqrt{\left(F_{i}^{X}\right)^{2}+\left(F_{i}^{Y}\right)^{2}}}\right) .
$$

Noting that $0 \leq \theta_{i}^{(1)} \leq \pi / 2$, we see that 1$)-1 \leq \Psi_{1} \leq 1$, 2) $\Psi_{1}=-1$ if and only if $F_{i}^{Y}=0$ and $F_{i}^{X}>0$ $(i=1, \cdots, R-1)$, and 3) $\Psi_{1}=1$ if and only if $F_{i}^{X}=0$ and $F_{i}^{Y}>0 \quad(i=1, \cdots, R-1)$. When the MH model holds, $\Psi_{1}$ equals zero. 


\subsection{Submeasure II}

Let

$$
S_{i}^{X}=1-F_{i}^{X}, S_{i}^{Y}=1-F_{i}^{Y} \text { for } i=1, \cdots, R-1 .
$$

The MH model may be expressed as

$$
S_{i}^{X}=S_{i}^{Y} \text { for } i=1, \cdots, R-1 .
$$

Let

$$
\Delta_{2}=\sum_{i=1}^{R-1}\left(S_{i}^{X}+S_{i}^{Y}\right)
$$

and for $i=1, \cdots, R-1$,

$$
\begin{gathered}
\theta_{i}^{(2)}=\sin ^{-1}\left(\frac{S_{i}^{X}}{\sqrt{\left(S_{i}^{X}\right)^{2}+\left(S_{i}^{Y}\right)^{2}}}\right) \\
S_{1(i)}^{*}=\frac{S_{i}^{X}}{\Delta_{2}}, \quad S_{2(i)}^{*}=\frac{S_{i}^{Y}}{\Delta_{2}} .
\end{gathered}
$$

Note that $\sum_{i=1}^{R-1}\left(S_{1(i)}^{*}+S_{2(i)}^{*}\right)=1$. Assuming that $\left\{S_{i}^{X}+S_{i}^{Y} \neq 0\right\}$, we shall define the submeasure $\Psi_{2}$ as follows;

$$
\Psi_{2}=\frac{4}{\pi} \sum_{i=1}^{R-1}\left(S_{1(i)}^{*}+S_{2(i)}^{*}\right)\left(\theta_{i}^{(2)}-\frac{\pi}{4}\right) .
$$

Noting that $0 \leq \theta_{i}^{(2)} \leq \pi / 2$, we see that 1 ) $-1 \leq \Psi_{2} \leq 1$; 2) $\Psi_{2}=-1$ if and only if $S_{i}^{X}=0$ and $S_{i}^{Y}>0 \quad(i=1, \cdots, R-1)$; and 3) $\Psi_{2}=1$ if and only if $S_{i}^{Y}=0$ and $S_{i}^{X}>0 \quad(i=1, \cdots, R-1)$. When the $\mathrm{MH}$ model holds, $\Psi_{2}$ equals zero.

\subsection{Complete Measure}

Assume that $\left\{F_{i}^{X}+F_{i}^{Y} \neq 0\right\}$ and $\left\{S_{i}^{X}+S_{i}^{Y} \neq 0\right\}$. Consider a measure defined by

$$
\Psi=\frac{1}{2}\left(\Psi_{1}+\Psi_{2}\right) \text {. }
$$

We see that 1) $-1 \leq \Psi \leq 1,2) \quad \Psi=-1$ if and only if $F_{i}^{X}=1$ (then $S_{i}^{X}=0$ ) and $F_{i}^{Y}=0$ (then $S_{i}^{Y}=1$ ) for all $i=1, \cdots, R-1$, and 3) $\Psi=1$ if and only if $F_{i}^{X}=0$ (then $S_{i}^{X}=1$ ) and $F_{i}^{Y}=1$ (then $S_{i}^{Y}=0$ ) for all $i=1, \cdots, R-1$. Thus, $\Psi=-1$ indicates that $p_{1 R}=1$ and the other cell probabilities are zero (say, upper-rightmarginal inhomogeneity), and $\Psi=1$ indicates that $p_{R 1}=1$ and the other cell probabilities are zero (say, lower-left-marginal inhomogeneity). When $\Psi=0$, we shall refer to this structure as the weak marginal homogeneity I (WMH-I). We note that if the MH model holds then the structure of WMH-I holds, but the converse does not hold.

Therefore, using the measure $\Psi$, we can see whether the structure of WMH-I departs toward the upper-rightmarginal inhomogeneity or toward the lower-left-marginal inhomogeneity. As the measure $\Psi$ approaches -1 , the departure from WMH-I becomes greater toward the upper-right-marginal inhomogeneity. While as the $\Psi$ approaches 1, it becomes greater toward the lower-leftmarginal inhomogeneity.

\section{Weak Marginal Homogeneity II and Measure}

Let

$$
\begin{aligned}
& T_{i}^{X}=\operatorname{Pr}(X \leq i \mid X \neq Y)=\sum_{k=1}^{i} p_{k \cdot}^{c}, \\
& T_{i}^{Y}=\operatorname{Pr}(Y \leq i \mid X \neq Y)=\sum_{k=1}^{i} p_{\cdot k}^{c}
\end{aligned}
$$

for $i=1, \cdots, R-1$, where

$$
p_{k \cdot}^{c}=\frac{1}{\delta}\left(p_{k \cdot}-p_{k k}\right), \quad p_{\cdot k}^{c}=\frac{1}{\delta}\left(p_{\cdot k}-p_{k k}\right), \delta=\sum \sum_{s \neq t} p_{s t} .
$$

The MH model may be expressed by

$$
T_{i}^{X}=T_{i}^{Y} \text { for } i=1, \cdots, R-1 .
$$

We shall consider the submeasure $\Upsilon_{1}$ which is defined by the submeasure $\Psi_{1}$ replaced $\left\{F_{i}^{X}\right\}$ and $\left\{F_{i}^{Y}\right\}$ by $\left\{T_{i}^{X}\right\}$ and $\left\{T_{i}^{Y}\right\}$, respectively.

Let

$$
U_{i}^{X}=1-T_{i}^{X}, U_{i}^{Y}=1-T_{i}^{Y} \text { for } i=1, \cdots, R-1 .
$$

The $\mathrm{MH}$ model may be expressed by

$$
U_{i}^{X}=U_{i}^{Y} \text { for } i=1, \cdots, R-1 .
$$

We shall consider the submeasure $\Upsilon_{2}$ which is defined by the submeasure $\Psi_{2}$ replaced $\left\{S_{i}^{X}\right\}$ and $\left\{S_{i}^{Y}\right\}$ by $\left\{U_{i}^{X}\right\}$ and $\left\{U_{i}^{Y}\right\}$, respectively.

Assume that $\left\{T_{i}^{X}+T_{i}^{Y} \neq 0\right\}$ and $\left\{U_{i}^{X}+U_{i}^{Y} \neq 0\right\}$. Consider a measure defined by

$$
\Upsilon=\frac{1}{2}\left(\Upsilon_{1}+\Upsilon_{2}\right)
$$

We see that $-1 \leq \Upsilon \leq 1$. Let $p_{i j}^{c}=p_{i j} / \delta \quad(i \neq j)$. In a similar way to $\Psi, \Upsilon=-1$ indicates that $p_{1 R}^{c}=1$ and the other $p_{i j}^{c}$ are zero $(i \neq j$ ) (say, conditional upperright-marginal inhomogeneity), and $\Upsilon=1$ indicates that $p_{R 1}^{c}=1$ and the other $p_{i j}^{c}$ are zero $(i \neq j)$ (say, conditional lower-left-marginal inhomogeneity). When $\Upsilon=0$, we shall refer to this structure as the weak marginal homogeneity II (WMH-II). We note that if the MH model holds then the structure of WMH-II holds, but the converse does not hold. 


\section{Relationships between Measures and Models}

We shall consider the relationship between the measure $\Psi$ (or $\Upsilon$ ) and the ML model. The ML model is given by

$$
L_{i}^{X}-L_{i}^{Y}=\Delta \text { for } i=1, \cdots, R-1,
$$

where

$$
L_{i}^{X}=\log \left(\frac{F_{i}^{X}}{1-F_{i}^{X}}\right), L_{i}^{Y}=\log \left(\frac{F_{i}^{Y}}{1-F_{i}^{Y}}\right) .
$$

A special case of this model obtained by putting $\Delta=0$ is the MH model. The ML model may also be expressed as

$$
F_{i}^{X}=\frac{\exp \left(\theta_{i}\right)}{1+\exp \left(\theta_{i}\right)}, \quad F_{i}^{Y}=\frac{\exp \left(\theta_{i}-\Delta\right)}{1+\exp \left(\theta_{i}-\Delta\right)},
$$

for $i=1, \cdots, R-1$. Therefore, when the ML model holds, 1) $\Delta>0$ if and only if $\left.\left\{F_{i}^{X}>F_{i}^{Y}\right\}, 2\right) \Delta<0$ if and only if $\left\{F_{i}^{X}<F_{i}^{Y}\right\}$, and 3) $\Delta=0$ if and only if $\left\{F_{i}^{X}=F_{i}^{Y}\right\}$. We obtain the following theorem.

Theorem 1. When the ML model holds,

1) $\Delta>0$ if and only if $\Psi<0 \quad(\Upsilon<0)$,

2) $\Delta<0$ if and only if $\Psi>0 \quad(\Upsilon>0)$,

3) $\Delta=0$ (i.e., the MH model holds) if and only if $\Psi=0 \quad(\Upsilon=0)$.

Next, we shall consider the relationship between the measure $\Psi$ (or $\Upsilon$ ) and the EMH model, defined by

$$
G_{1(i)}=\tau G_{2(i)} \text { for } i=1, \cdots, R-1,
$$

where $G_{1(i)}=\sum_{s=1}^{i} \sum_{t=i+1}^{R} p_{s t}, G_{2(i)}=\sum_{s=i+1}^{R} \sum_{t=1}^{i} p_{s t}$.

A special case of this model obtained by putting $\tau=1$ is the MH model. Noting that $G_{1(i)}-G_{2(i)}=F_{i}^{X}-F_{i}^{Y}$ ( $i=1, \cdots, R-1)$, we obtain the following theorem.

Theorem 2. When the EMH model holds,

1) $\tau>1$ if and only if $\Psi<0 \quad(\Upsilon<0)$,

2) $\tau<1$ if and only if $\Psi>0 \quad(\Upsilon>0)$,

3) $\tau=1$ (i.e., the MH model holds) if and only if $\Psi=0 \quad(\Upsilon=0)$.

Thus, when the ML (EMH) model holds, the measures $\Psi$ and $\Upsilon$ are adequate to represent the degree of departure from $\mathrm{MH}$.

\section{Approximate Confidence Interval for Measures}

Let $n_{i j}$ denote the observed frequency in the $i$ th row and $j$ th column of the table $(i=1, \cdots, R ; j=1, \cdots, R)$. Assuming that a multinomial distribution applies to the $R \times R$ table, we shall consider an approximate standard error and large-sample confidence interval for the measure $\Psi$, using the delta method, as described by [8]. The sample version of $\Psi$, i.e., $\hat{\Psi}$, is given by $\Psi$ with $\left\{p_{i j}\right\}$ replaced by $\left\{\hat{p}_{i j}\right\}$, where $\hat{p}_{i j}=n_{i j} / n$ and $n=\sum \sum n_{i j}$. Using the delta method, we obtain the following theorem.

Theorem 3. $\sqrt{n}(\hat{\Psi}-\Psi)$ has asymptotically (as $n \rightarrow \infty)$ a normal distribution with mean zero and variance $\sigma^{2}[\hat{\Psi}]$, where

$$
\sigma^{2}[\hat{\Psi}]=\frac{1}{4} \sum_{i=1}^{R} \sum_{j=1}^{R}\left(a_{i j}+b_{i j}\right)^{2} p_{i j},
$$

with $a_{i j}=\frac{4}{\pi \Delta_{1}} \sum_{k=1}^{R-1}\left[\{I(i \leq k)+I(j \leq k)\} \theta_{k}^{(1)}+\frac{F_{k}^{X}+F_{k}^{Y}}{\left(F_{k}^{X}\right)^{2}+\left(F_{k}^{Y}\right)^{2}}\left\{-I(i \leq k) F_{k}^{Y}+I(j \leq k) F_{k}^{X}\right\}\right]-\frac{\{2 R-(i+j)\}\left(\Psi_{1}+1\right)}{\Delta_{1}}$,

$$
b_{i j}=\frac{4}{\pi \Delta_{2}} \sum_{k=1}^{R-1}\left[\{I(i>k)+I(j>k)\} \theta_{k}^{(2)}+\frac{S_{k}^{X}+S_{k}^{Y}}{\left(S_{k}^{X}\right)^{2}+\left(S_{k}^{Y}\right)^{2}}\left\{I(i>k) S_{k}^{Y}-I(j>k) S_{k}^{X}\right\}\right]-\frac{\{(i+j)-2\}\left(\Psi_{2}+1\right)}{\Delta_{2}},
$$

and $I(\cdot)$ is the indicator function, $I(\cdot)=1$ if true, 0 if not.

Also, the sample version of $\Upsilon$, i.e., $\hat{\Upsilon}$, is given by $\Upsilon$ with $\left\{p_{i j}\right\}$ replaced by $\left\{\hat{p}_{i j}\right\}$. We obtain the following theorem.

Theorem 4. $\sqrt{n}(\hat{\Upsilon}-\Upsilon)$ has asymptotically (as

$n \rightarrow \infty)$ a normal distribution with mean zero and variance $\sigma^{2}[\hat{\Upsilon}]$, where

$$
\sigma^{2}[\hat{\Upsilon}]=\frac{1}{4} \sum_{i=1}^{R} \sum_{\substack{j=1 \\ j \neq i}}^{R}\left(c_{i j}+d_{i j}\right)^{2} p_{i j},
$$

with

$$
\begin{aligned}
C_{i j}= & \frac{4}{\delta \pi \Delta_{3}} \sum_{k=1}^{R-1}\left[\left\{\left(I(i \leq k)-T_{k}^{X}\right)+\left(I(j \leq k)-T_{k}^{Y}\right)\right\}\left(\theta_{k}^{(3)}-\frac{\pi}{4}\right)+\frac{T_{k}^{X}+T_{k}^{Y}}{\left(T_{k}^{X}\right)^{2}+\left(T_{k}^{Y}\right)^{2}}\left\{-\left(I(i \leq k)-T_{k}^{X}\right) T_{k}^{Y}+\left(I(j \leq k)-T_{k}^{Y}\right) T_{k}^{X}\right\}\right] \\
& -\frac{1}{\delta \Delta_{3}} \sum_{k=1}^{R-1}\left[\left(I(i \leq k)-T_{k}^{X}\right)+\left(I(j \leq k)-T_{k}^{Y}\right)\right] \Upsilon_{1},
\end{aligned}
$$




$$
\begin{aligned}
d_{i j}= & \frac{4}{\delta \pi \Delta_{4}} \sum_{k=1}^{R-1}\left[\left\{\left(I(i>k)-U_{k}^{X}\right)+\left(I(j>k)-U_{k}^{Y}\right)\right\}\left(\theta_{k}^{(4)}-\frac{\pi}{4}\right)+\frac{U_{k}^{X}+U_{k}^{Y}}{\left(U_{k}^{X}\right)^{2}+\left(U_{k}^{Y}\right)^{2}}\left\{\left(I(i>k)-U_{k}^{X}\right) U_{k}^{Y}-\left(I(j>k)-U_{k}^{Y}\right) U_{k}^{X}\right\}\right] \\
& -\frac{1}{\delta \Delta_{4}} \sum_{k=1}^{R-1}\left[\left(I(i>k)-U_{k}^{X}\right)+\left(I(j>k)-U_{k}^{Y}\right)\right] \Upsilon_{2},
\end{aligned}
$$$$
\Delta_{3}=\sum_{i=1}^{R-1}\left(T_{i}^{X}+T_{i}^{Y}\right), \quad \Delta_{4}=\sum_{i=1}^{R-1}\left(U_{i}^{X}+U_{i}^{Y}\right),
$$$$
\theta_{i}^{(3)}=\sin ^{-1}\left(\frac{T_{i}^{Y}}{\sqrt{\left(T_{i}^{X}\right)^{2}+\left(T_{i}^{Y}\right)^{2}}}\right),
$$$$
\theta_{i}^{(4)}=\sin ^{-1}\left(\frac{U_{i}^{X}}{\sqrt{\left(U_{i}^{X}\right)^{2}+\left(U_{i}^{Y}\right)^{2}}}\right) .
$$

inhomogeneity which indicates that the grade of right eye for arbitrary woman is "Best" and the grade of her left eye is "Worst".

Example 2: Consider the unaided vision data in Table 1(b), taken from [9]. We see from Table 2 that for the

Table 1. Unaided distance vision data of (a) 7477 women in Britain from [1]; (b) 3242 men in Britain from [9] and (c) 4746 students in Japan from [3].

(a) Women in Britain

Let $\hat{\sigma}^{2}[\hat{\Psi}]$ denote $\sigma^{2}[\hat{\Psi}]$ with $\left\{p_{i j}\right\}$ replaced by $\left\{\hat{p}_{i j}\right\}$. Then $\hat{\sigma}[\hat{\Psi}] / \sqrt{n}$ is estimated approximate standard error for $\hat{\Psi}$, and $\hat{\Psi} \pm z_{p / 2} \hat{\sigma}[\hat{\Psi}] / \sqrt{n}$ is approximate $100(1-p)$ percent confidence interval for $\Psi$, where $z_{p / 2}$ is the percentage point from the standard normal distribution corresponding to a two-tail probability equal to $p$. We also obtain the similar result for measure $\Upsilon$.

\section{Examples}

Example 1: Consider the unaided distance vision data in Table 1(a) taken from [1]. There are data on unaided distance vision of 7477 women aged 30 to 39 employed in Royal Ordnance factories in Britain from 1943 to 1946. We see from Table 2 that for the data in Table 1(a), the estimated value of the measure $\Psi$ is -0.0130 and all values in confidence interval for $\Psi$ are negative. Therefore, the structure of WMH-I for a woman's right and left eyes departs toward the upper-right-marginal inhomogeneity. Also we see from Table 3 that for the data in Table 1(a), the estimated value of the measure $\Upsilon$ is -0.0436 and all values in confidence interval for $\Upsilon$ are negative. Therefore, the structure of WMH-II for a woman's right and left eyes departs toward the conditional upper-right-marginal inhomogeneity.

Table 4 gives the values of likelihood ratio chi-squared statistic for testing goodness-of-fit of each of MH, ML, and EMH models. We see from Table 4 that each of ML and EMH models fits these data well. Thus the measures $\Psi$ and $\Upsilon$ would indicate the degree of departure from $\mathrm{MH}$. We can see from these measures that the degree of departure from $\mathrm{MH}$ for the vision data in Table 1(a) is estimated to be 1.30 (4.36) percent of the maximum

\begin{tabular}{|c|c|c|c|c|c|c|}
\hline \multirow{2}{*}{\multicolumn{2}{|c|}{$\begin{array}{l}\text { Right eye } \\
\text { grade }\end{array}$}} & \multicolumn{4}{|c|}{ Left eye grade } & \multirow[b]{2}{*}{ Total } \\
\hline & & (1) & (2) & \multirow{2}{*}{$\frac{(3)}{124}$} & $(4)$ & \\
\hline \multicolumn{2}{|l|}{ Best (1) } & 1520 & 266 & & 66 & 1976 \\
\hline \multicolumn{2}{|c|}{ Second (2) } & 234 & 1512 & 432 & 78 & 2256 \\
\hline \multicolumn{2}{|c|}{ Third (3) } & 117 & 362 & 1772 & 205 & 2456 \\
\hline \multicolumn{2}{|c|}{ Worst (4) } & 36 & 82 & 179 & 492 & 789 \\
\hline \multicolumn{2}{|l|}{ Total } & 1907 & 2222 & 2507 & 841 & 7477 \\
\hline \multicolumn{7}{|c|}{ (b) Men in Britain } \\
\hline \multirow{2}{*}{\multicolumn{2}{|c|}{$\begin{array}{c}\text { Right eye } \\
\text { grade }\end{array}$}} & \multicolumn{4}{|c|}{ Left eye grade } & \\
\hline & & (1) & (2) & (3) & (4) & Total \\
\hline \multicolumn{2}{|l|}{ Best (1) } & 821 & 112 & 85 & 35 & 1053 \\
\hline \multicolumn{2}{|l|}{ Second (2) } & 116 & 494 & 145 & 27 & 782 \\
\hline \multicolumn{2}{|l|}{ Third (3) } & 72 & 151 & 583 & 87 & 893 \\
\hline \multicolumn{2}{|l|}{ Worst (4) } & 43 & 34 & 106 & 331 & 514 \\
\hline \multicolumn{2}{|l|}{ Total } & 1052 & 791 & 919 & 480 & 3242 \\
\hline \multicolumn{7}{|c|}{ (c) Students in Japan } \\
\hline \multirow{2}{*}{$\begin{array}{c}\text { Right eye } \\
\text { grade }\end{array}$} & \multicolumn{5}{|c|}{ Left eye grade } & \\
\hline & (1) & (2) & & (3) & (4) & Total \\
\hline Best (1) & 1291 & 130 & & 40 & 22 & 1483 \\
\hline Second (2) & 149 & 221 & & 114 & 23 & 507 \\
\hline Third (3) & 64 & 124 & & 660 & 185 & 1033 \\
\hline Worst (4) & 20 & 25 & & 249 & 1429 & 1723 \\
\hline Total & 1524 & 500 & & 1063 & 1659 & 4746 \\
\hline
\end{tabular}
departure toward the (conditional) upper-right-marginal

Table 2. Estimates of $\Psi$, estimated approximate standard errors for $\hat{\Psi}$, and approximate $95 \%$ confidence intervals for $\Psi$, applied to Table 1 .

\begin{tabular}{cccc}
\hline Table & $\hat{\Psi}$ & S. E. & C. I. \\
\hline 1(a) & -0.0130 & 0.0037 & $(-0.0203,-0.0056)$ \\
$1(\mathrm{~b})$ & 0.0055 & 0.0064 & $(-0.0071,0.0181)$ \\
$1(\mathrm{c})$ & 0.0125 & 0.0040 & $(0.0048,0.0203)$ \\
\hline
\end{tabular}


Table 3. Estimates of $\Upsilon$, estimated approximate standard errors for $\hat{\Upsilon}$, and approximate $95 \%$ confidence intervals for $\Upsilon$, applied to Table 1 .

\begin{tabular}{cccc}
\hline Table & $\hat{\Upsilon}$ & S. E. & C. I. \\
\hline $1(a)$ & -0.0436 & 0.0126 & $(-0.0683,-0.0190)$ \\
$1(\mathrm{~b})$ & 0.0172 & 0.0201 & $(-0.0222,0.0566)$ \\
$1(\mathrm{c})$ & 0.0517 & 0.0163 & $(0.0198,0.0836)$ \\
\hline
\end{tabular}

Table 4. Values of likelihood ratio chi-squared statistic for the MH, ML, and EMH models applied to Table 1.

\begin{tabular}{ccccc}
\hline $\begin{array}{c}\text { Applied } \\
\text { models }\end{array}$ & $\begin{array}{c}\text { Degrees of } \\
\text { freedom }\end{array}$ & 1(a) & Table \\
\hline MH & 3 & $11.99^{*}$ & 3.68 & $11.18^{*}$ \\
ML & 2 & 0.39 & 3.16 & 1.41 \\
EMH & 2 & 0.005 & 2.94 & 0.56 \\
\hline
\end{tabular}

*Means significant at the 0.05 level.

data in Table 1(b) the estimated value of measure $\Psi$ is 0.0055 and the confidence interval for $\Psi$ includes zero. So this may indicate that there is a structure of WMH-I in the data in Table 1(b). Also we see from Table 3 that for the data in Table 1(b), the estimated value of measure $\Upsilon$ is 0.0172 and the confidence interval for $\Upsilon$ includes zero. So this may indicate that there is a structure of WMH-II in the data in Table 1(b).

Example 3: Consider the data in Table 1(c) taken from $[3,10]$. We see from Table 2 that for the data in Table 1(c), the estimated value of the measure $\Psi$ is 0.0125 and all values in confidence interval for $\Psi$ are positive. Therefore, the structure of WMH-I for a student's right and left eyes departs toward the lower-leftmarginal inhomogeneity. Also we see from Table 3 that for the data in Table 1(c), the estimated value of the measure $\Upsilon$ is 0.0517 and all values in confidence interval for $\Upsilon$ are positive. Therefore, the structure of WMH-II for a student's right and left eyes departs toward the conditional lower-left-marginal inhomogeneity.

We see from Table 4 that each of ML and EMH models fits these data well. Thus the measures $\Psi$ and $\Upsilon$ would indicate the degree of departure from $\mathrm{MH}$. We can see from these measures that the degree of departure from MH for the vision data in Table 1(c) is estimated to be 1.25 (5.17) percent of the maximum departure toward the (conditional) lower-left-marginal inhomogeneity which indicates that the grade of right eye for arbitrary student is "Worst" and the grade of his/her left eye is "Best".

\section{Concluding Remarks}

For the analysis of square contingency tables with ordered categories, when the ML model, or the EMH model, or other asymmetry models, for example, [11]'s conditional symmetry model (defined by $p_{i j} / p_{j i}=\theta$ for $i<j$ ) holds, the proposed measures $\Psi$ and $\Upsilon$ are adequate to represent the degree of departure from the MH model toward two maximum departures, i.e., toward the (conditional) lower-left-marginal inhomogeneity or toward the (conditional) upper-right-marginal inhomogeneity.

\section{Discussion}

$[6,7]$ considered the measures to represent the degree of departure from MH. The present paper has considered two types of maximum marginal inhomogeneity (i.e., the lower-left-marginal inhomogeneity and the upper-rightmarginal inhomogeneity). The measures in $[6,7]$ take the value 1 in two types of maximum marginal inhomogeneity. The measures $\Psi$ and $\Upsilon$ in the present paper can distinguish these two kinds of maximum marginal inhomogeneity by the values -1 or 1 although the measures in $[6,7]$ cannot distinguish them. Also the proposed measures can represent the degree of departure from $\mathrm{MH}$ when the ML or the EMH models, or the other asymmetry models hold. Therefore for the ordinal data, the proposed measures rather than those in [6,7] may be useful to represent the degree of departure from $\mathrm{MH}$.

\section{Acknowledgements}

The authors would like to express their sincere thanks to the editor and a referee for their helpful comments.

\section{REFERENCES}

[1] A. Stuart, "A Test for Homogeneity of the Marginal Distributions in a Two-Way Classification," Biometrika, Vol. 42, No. 3-4, 1955, pp. 412-416. doi:10.1093/biomet/42.3-4.412

[2] A. Agresti, "Analysis of Ordinal Categorical Data," John Wiley, New York, 1984.

[3] S. Tomizawa, "Three Kinds of Decompositions for the Conditional Symmetry Model in a Square Contingency Table," Journal of the Japan Statistical Society, Vol. 14, No. 1, 1984, pp. 35-42.

[4] S. Tomizawa, "A Generalization of the Marginal Homogeneity Model for Square Contingency Tables with Ordered Categories," Journal of Educational and Behavioral Statistics, Vol. 20, 1995, pp. 349-360.

[5] K. Tahata and S. Tomizawa, "Generalized Marginal Homogeneity Model and Its Relation to Marginal Equimoments for Square Contingency Tables with Ordered Categories," Advances in Data Analysis and Classification, Vol. 2, No. 3, 2008, pp. 295-311. doi:10.1007/s11634-008-0028-1

[6] K. Tahata, T. Iwashita and S. Tomizawa, "Measure of Departure from Symmetry of Cumulative Marginal Probabilities for Square Contingency Tables with Ordered Categories," SUT Journal of Mathematics, Vol. 42, 2006, 
pp. 7-29.

[7] K. Tahata, T. Iwashita and S. Tomizawa, "Measure of Departure from Conditional Marginal Homogeneity for Square Contingency Tables with Ordered Categories," Statistics, Vol. 42, No. 5, 2008, pp. 453-466. doi:10.1080/02331880802190521

[8] Y. M. M. Bishop, S. E. Fienberg and P. W. Holland, "Discrete Multivariate Analysis: Theory and Practice," The MIT Press, Cambridge, 1975.

[9] A. Stuart, "The Estimation and Comparison of Strengths of Association in Contingency Tables," Biometrika, Vol.
40, 1953, pp. 105-110. doi:10.2307/2333101

[10] K. Yamamoto, K. Tahata, N. Miyamoto and S. Tomizawa, "Comparison between Several Square Tables Data Using Models of Symmetry and Asymmetry," Quantum Probability and White Noise Analysis: Quantum Bio-Informatics, Vol. 21, 2007, pp. 337-349.

[11] P. McCullagh, "A Class of Parametric Models for the Analysis of Square Contingency Tables with Ordered Categories," Biometrika, Vol. 65, No. 2, 1978, pp. 413418. doi:10.1093/biomet/65.2.413 